\title{
All-in-one adeno-associated virus delivery and genome editing by Neisseria meningitidis Cas9 in vivo
}

\author{
Raed Ibraheim ${ }^{1}$, Chun-Qing Song ${ }^{1}$, Aamir Mir ${ }^{1}$, Nadia Amrani ${ }^{1}$, Wen Xue ${ }^{1,2}$ and Erik J. Sontheimer ${ }^{1,2^{*}}$ (D)
}

\begin{abstract}
Background: Clustered, regularly interspaced, short palindromic repeats (CRISPR) and CRISPR-associated proteins (Cas) have recently opened a new avenue for gene therapy. Cas9 nuclease guided by a single-guide RNA (sgRNA) has been extensively used for genome editing. Currently, three Cas9 orthologs have been adapted for in vivo genome engineering applications: Streptococcus pyogenes Cas9 (SpyCas9), Staphylococcus aureus Cas9 (SauCas9), and Campylobacter jejuni (CjeCas9). However, additional in vivo editing platforms are needed, in part to enable a greater range of sequences to be accessed via viral vectors, especially those in which Cas9 and sgRNA are combined into a single vector genome.

Results: Here, we present in vivo editing using Neisseria meningitidis Cas9 (NmeCas9). NmeCas9 is compact, edits with high accuracy, and possesses a distinct protospacer adjacent motif (PAM), making it an excellent candidate for safe gene therapy applications. We find that NmeCas9 can be used to target the Pcsk9 and Hpd genes in mice. Using tail-vein hydrodynamicbased delivery of NmeCas9 plasmid to target the Hpd gene, we successfully reprogram the tyrosine degradation pathway in Hereditary Tyrosinemia Type I mice. More importantly, we deliver NmeCas9 with its sgRNA in a single recombinant adeno-associated vector (rAAV) to target Pcsk9, resulting in lower cholesterol levels in mice. This all-in-one vector yielded $>35 \%$ gene modification after two weeks of vector administration, with minimal off-target cleavage in vivo.
\end{abstract}

Conclusions: Our findings indicate that NmeCas9 can enable the editing of disease-causing loci in vivo, expanding the targeting scope of RNA-guided nucleases.

Keywords: CRISPR, All-in-one rAAV, Genome editing, NmeCas9

\section{Background}

A major advance in the field of gene therapy has been the introduction of Cas9 nuclease-enabled genome editing [1]. Clustered, regularly interspaced, short palindromic repeats (CRISPR) loci specify an adaptive immune pathway that evolved in bacteria and archaea to defend against mobile genetic elements (MGEs) [2, 3]. The effector complex in type II CRISPR systems includes the Cas9 nuclease, which is guided by a CRISPR RNA (crRNA) and a trans-activating RNA (tracrRNA). These dual RNAs can be fused to form a single-guide RNA (sgRNA) [4]. Each crRNA contains a unique "spacer" sequence that can be programmed to cleave a DNA

\footnotetext{
* Correspondence: erik.sontheimer@umassmed.edu

${ }^{1}$ RNA Therapeutics Institute, University of Massachusetts Medical School, Worcester, MA 01605, USA

${ }^{2}$ Program in Molecular Medicine, University of Massachusetts Medical School, Worcester, MA 01605, USA
}

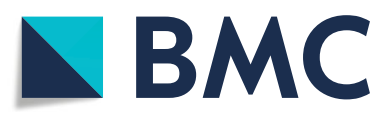

(c) The Author(s). 2018 Open Access This article is distributed under the terms of the Creative Commons Attribution 4.0 International License (http://creativecommons.org/licenses/by/4.0/), which permits unrestricted use, distribution, and

reproduction in any medium, provided you give appropriate credit to the original author(s) and the source, provide a link to the Creative Commons license, and indicate if changes were made. The Creative Commons Public Domain Dedication waiver (http://creativecommons.org/publicdomain/zero/1.0/) applies to the data made available in this article, unless otherwise stated. segment of interest. Cas9 scans DNA for a specific protospacer adjacent motif (PAM), opens the duplex to form an RNA-DNA hybrid between the guide and the spacer, and introduces a double-strand break (DSB) in the DNA target $[1,3]$. Cas9 and sgRNA have been adapted to enable genome editing in cultured cells following various modes of delivery including plasmid and RNA transfections, viral transduction, and ribonucleoprotein (RNP) electroporation. Precise and efficient in vivo editing is more difficult to achieve, largely due to the difficulties inherent in delivery.

Several methods have been developed to deliver Cas9 in vivo including viral and non-viral methods [5]. These include the use of gold and lipid nanoparticles to deliver Cas9 in RNP or RNA form in mice. However, these methods present challenges for routine use including cost and tissue distribution [6-8]. One of the more intriguing gene delivery vehicles that has emerged in recent years is 
recombinant adeno-associated virus (rAAV). This vector possesses several attributes that benefit gene therapy applications, including lack of pathogenicity and replication as well as an ability to infect dividing and non-dividing cells [9]. In addition, rAAV is also capable of infecting a wide range of cells and maintain sustained expression [10, 11]. Compared to other viral vectors, rAAV persists in concatemeric, episomal forms, while eliciting mild immune responses [12-14]. The usefulness of rAAV-based delivery for gene therapy is reflected in the number of clinical trials involving rAAV [15]. One of the most exciting advancements for rAAV gene therapy field has been the FDA's recent market approval of a therapy for RPE65-mediated inherited retinal disease (IRD), the first of its kind in the United States [16].

More recently, several groups have focused their efforts on using this tool for in vivo delivery of Cas9 orthologs [17-20]. The majority of Cas9 genome editing efforts have been focused on the widely used type II-A ortholog from Streptococcus pyogenes, SpyCas9. Although it exhibits consistently robust genome-editing activity, considerable effort has been required to overcome off-target editing activities of wild-type SpyCas9 [21-23] [Amrani et al., manuscript in revision (https:// www.biorxiv.org/content/early/2018/05/09/172650)]. Furthermore, its large size (1368 amino acids, $4.10 \mathrm{~kb}$ ) restricts its delivery with its guide in a single virion with potent vectors such as rAAV [24]. Split SpyCas9 constructs (expressed from separate viruses) have been employed [19], though activity is sometimes compromised [25-27]. Dual-rAAV delivery of SpyCas9 and sgRNA can be achieved [28], but it requires the usage of highly minimized promoters that limit expression and tissue specificity. Furthermore, dual rAAV formats carry significant costs as well as limitations in co-transduction.

Alternatively, compact Cas9 orthologs can be packaged in all-in-one rAAV vectors. Type II-A Staphylococcus aureus (SauCas9) (1053 amino acids, $3.16 \mathrm{~kb}$ ) and type II-C Campylobacter jejuni Cas9 (CjeCas9) (984 amino acids, $2.95 \mathrm{~kb}$ ) have been deployed successfully via rAAV in mice $[18,20]$. However, unlike the highly abundant NGG SpyCas9 PAM, these Cas9 nucleases have more restrictive PAM requirements (for SauCas9, 5'-NNGRRT-3'; for CjeCas9, 5'-N $\mathrm{N}_{4} \mathrm{RYAC}$ [29]. Furthermore, off-target editing by SauCas9 is not unusual $[18,30]$. For these reasons, many genomic sites of interest cannot be targeted by all-in-one rAAV delivery of the Cas9 genome editing machinery and additional capabilities and PAM specificities are therefore needed.

We and others have reported genome editing in mammalian cells by the type II-C Cas9 from Neisseria meningitidis strain 8013 (NmeCas9) [31-33] (Amrani et al., manuscript in revision). NmeCas9 is small (1082 amino acids, $3.16 \mathrm{~kb})$, targets PAMs $\left(\mathrm{N}_{4} \mathrm{GAYT}>\mathrm{N}_{4} \mathrm{GYTT} /\right.$
$\mathrm{N}_{4}$ GAYA/ $\mathrm{N}_{4} \mathrm{GTCT}$ ) that are distinct from those of the other compact Cas9 orthologs described above, and is intrinsically resistant to off-targeting [32] (Amrani et al., manuscript in revision). Additionally, NmeCas9 can be subjected to off-switch control by anti-CRISPR proteins [34], which could facilitate spatial and temporal control over NmeCas9 activity in vivo and ex vivo.

In this study, we report the in vivo delivery of NmeCas 9 and its guide by a single expression cassette that is sufficiently small for all-in-one rAAV vectors. Two disease genes were targeted separately to highlight the therapeutic potential of NmeCas9: the Hpd gene in a hereditary tyrosinemia type I (HTI) mouse model $\left(F a h^{\text {neo }}\right)$; and the Pcsk9 gene in $\mathrm{C} 57 \mathrm{Bl} / 6$ mice. Hpd encodes the 4-hydroxyphenylpyruvate dioxygenase enzyme in the tyrosine metabolism pathway and disrupting $H p d$ can lead to a decrease in the accumulation of toxic fumarylacetoacetate in tyrosinemia models [35]. Separately, Pcsk 9 encodes proprotein convertase subtilisin/kexin type 9 (PCSK9), an antagonist of the low-density lipoprotein (LDL) receptor [36, 37]. When PCSK9 is knocked out, more LDL receptors are available at the surface of hepatocytes to allow cholesterol binding and recycling towards the lysosomes for degradation $[38,39]$. The alleviation of tyrosinemia symptoms upon $H p d$ disruption, as well as the reduced serum cholesterol levels that result from Pcsk 9 disruption, provide convenient readouts and benchmarks for genome editing activity $[18,35]$. We used these systems to validate all-in-one rAAV delivery of NmeCas9 as an effective in vivo genome editing platform in mammals.

\section{Results}

Efficient genome editing using all-in-one AAV-sgRNAhNmeCas9 plasmid in cells and in vivo by hydrodynamic injection

Recently, we have shown that the relatively compact NmeCas9 is active in genome editing in a range of cell types (Amrani et al., manuscript in revision). To exploit the small size of this Cas9 ortholog, we generated an all-in-one AAV construct with human-codon-optimized NmeCas9 under the expression of the mouse U1a promoter and with its sgRNA driven by the U6 promoter (Fig. 1a).

Two sites in the mouse genome were selected initially to test the nuclease activity of NmeCas9 in vivo: the Rosa26 "safe-harbor" gene (targeted by sgRosa26); and the proprotein convertase subtilisin/kexin type 9 (Pcsk9) gene (targeted by $\operatorname{sg} P c s k 9$ ), a common therapeutic target for lowering circulating cholesterol and reducing the risk of cardiovascular disease (Fig. 1b). Genome-wide off-target predictions for these guides were determined computationally using the Bioconductor package CRISPRseek 1.9.1 [40] with $\mathrm{N}_{4} \mathrm{GN}_{3}$ PAMs and up to six mismatches. Many $\mathrm{N}_{4} \mathrm{GN}_{3}$ PAMS are inactive, so these search parameters are nearly certain to cast a wider net than the true off-target 

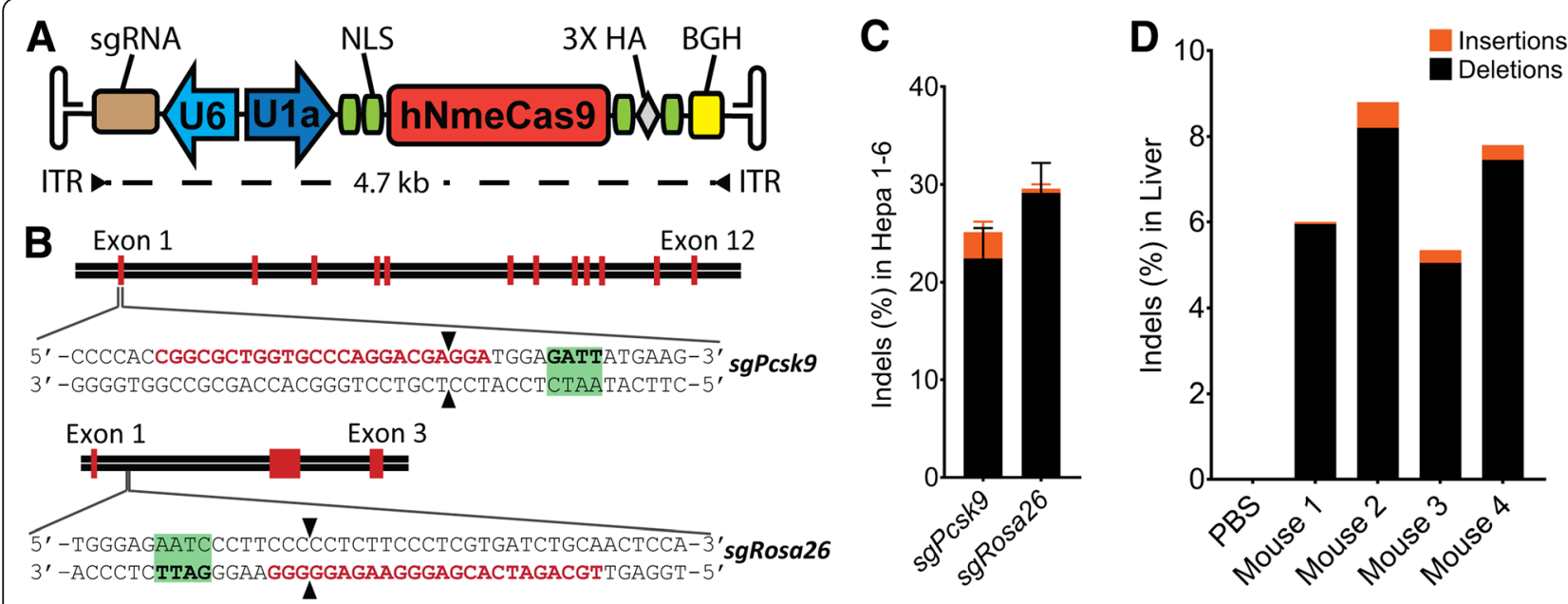

Fig. 1 Validation of an all-in-one AAV-sgRNA-hNmeCas9 construct. a Schematic representation of a single rAAV vector expressing human-codonoptimized NmeCas 9 and its sgRNA. The backbone is flanked by AAV inverted terminal repeats (ITR). The poly(a) signal is from rabbit beta-globin (BGH). b Schematic diagram of the Pcsk9 (top) and Rosa26 (bottom) mouse genes. Red bars represent exons. Zoomed-in views show the protospacer sequence (red) whereas the NmeCas9 PAM sequence is highlighted in green. Double-stranded break location sites are denoted (black arrowheads). c Stacked histogram showing the percentage distribution of insertions-deletions (indels) obtained by TIDE after AAV-sgRNA-hNmeCas9 plasmid transfections in Hepa1-6 cells targeting Pcsk9 (sgPcsk9) and Rosa26 (sgRosa26) genes. Data are presented as mean values \pm SD from three biological replicates. d Stacked histogram showing the percentage distribution of indels at Pcsk9 in the liver of C57BI/6 mice obtained by TIDE after hydrodynamic injection of AAV-sgRNA-hNmeCas9 plasmid

profile. Despite the expansive nature of the search, our analyses revealed no off-target sites with fewer than four mismatches in the mouse genome (Additional file 1: Figure S1). On-target editing efficiencies at these target sites were evaluated in mouse Hepa1-6 hepatoma cells by plasmid transfections and indel quantification was performed by sequence trace decomposition using the Tracking of Indels by Decomposition (TIDE) web tool [41]. We found $>25 \%$ indel values for the selected guides, the majority of which were deletions (Fig. 1c).

To evaluate the preliminary efficacy of the constructed all-in-one AAV-sgRNA-hNmeCas9 vector, endotoxin-free sgPcsk9 plasmid was hydrodynamically administered into the $\mathrm{C} 57 \mathrm{Bl} / 6$ mice via tail-vein injection. This method can deliver plasmid DNA to $\sim 40 \%$ of hepatocytes for transient expression [42]. Indel analyses by TIDE using DNA extracted from liver tissues revealed 5-9\% indels 10 days after vector administration (Fig. 1d), comparable to the editing efficiencies obtained with analogous tests of SpyCas9 [43]. These results suggested that NmeCas9 is capable of editing liver cells in vivo.

\section{Knockout of 4-Hydroxyphenylpyruvate dioxygenase rescues the lethal phenotypes of hereditary Tyrosinemia type I mice}

Hereditary Tyrosinemia type I (HT-I) is a fatal genetic disease caused by autosomal recessive mutations in the Fah gene, which codes for the fumarylacetoacetate hydroxylase (FAH) enzyme. Patients with diminished FAH have a disrupted tyrosine catabolic pathway, leading to the accumulation of toxic fumarylacetoacetate and succinyl acetoacetate, causing liver and kidney damage [44]. Over the past two decades, the disease has been controlled by 2-(2-Nitro-4-trifluoromethylbenzoyl) -1,3- cyclohexanedione (NTBC), which inhibits 4-hydroxyphenylpyruvate dioxygenase upstream in the tyrosine degradation pathway, thus preventing the accumulation of the toxic metabolites [45]. However, this treatment requires lifelong management of diet and medication and may eventually require liver transplantation [46].

Several gene therapy strategies have been tested to correct the defective Fah gene using site-directed mutagenesis [47] or homology-directed repair by CRISPR-Cas9 [47-49]. It has been reported that successful modification of only $1 /$ 10,000 of hepatocytes in the liver is sufficient to rescue the phenotypes of Fah ${ }^{\text {mut/mut }}$ mice. Recently, a metabolic pathway reprogramming approach has been suggested in which the function of the hydroxyphenylpyruvate dioxygenase (HPD) enzyme was disrupted by the deletion of exons 3 and 4 of the Hpd gene in the liver [35]. This provides us with a context in which to test the efficacy of NmeCas9 editing, by targeting Hpd and assessing rescue of the disease phenotype in Fah mutant mice [50]. For this purpose, we screened and identified two target sites (one each in exon 8 [sgHpd1] and exon 11 [sgHpd2]) within the open reading frame of $\mathrm{Hpd}$ (Fig. 2a). These guides induced average indel efficiencies of $10.8 \%$ and $9.1 \%$, respectively, by plasmid transfections in Hepa1-6 cells (Additional file 1: Figure S2). 


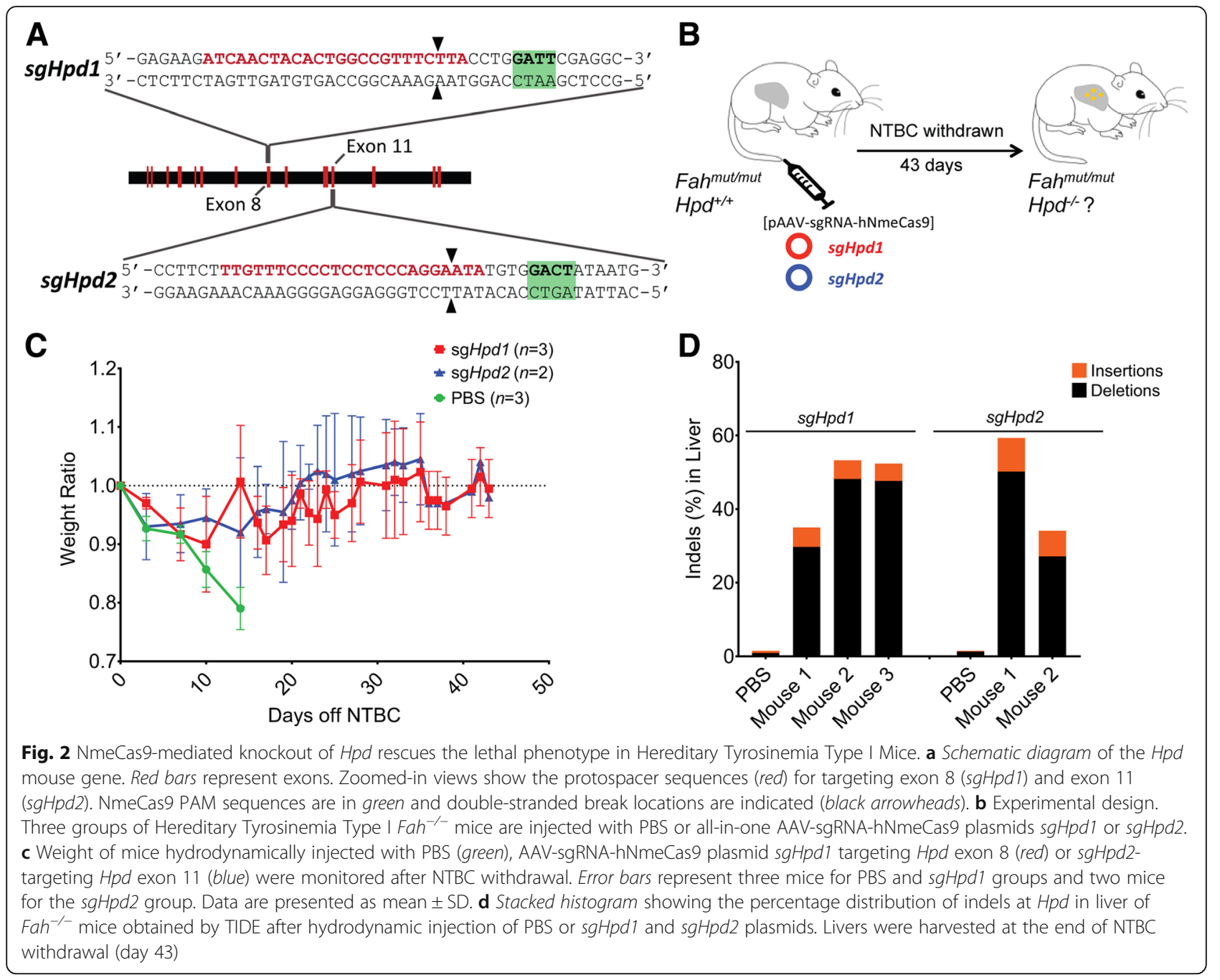

Three groups of mice were treated by hydrodynamic injection with either phosphate-buffered saline (PBS) or with one of the two sgHpd1 and sgHpd2 all-in-one AAV-sgRNA-hNmeCas9 plasmids. One mouse in the sgHpd1 group and two in the sgHpd2 group were excluded from the follow-up study due to failed tail-vein injections. Mice were taken off NTBC-containing water seven days after injections and their weight was monitored for 43 days post injection (Fig. 2b). Mice injected with PBS suffered severe weight loss (a hallmark of HT-I) and were sacrificed after losing $20 \%$ of their body weight (Fig. 2c). Overall, all $s g H p d 1$ and $s g H p d 2$ mice successfully maintained their body weight for 43 days overall and for at least 21 days without NTBC (Fig. 2c). NTBC treatment had to be resumed for 2-3 days for two mice that received sgHpd1 and one that received sgHpd2 to allow them to regain body weight during the third week after plasmid injection, perhaps due to low initial editing efficiencies, liver injury due to hydrodynamic injection, or both. Conversely, all other sgHpd1 and sgHpd2 treated mice achieved indels with frequencies in the range of $35-60 \%$ (Fig. 2d). This level of gene inactivation likely reflects not only the initial editing events but also the competitive expansion of edited cell lineages (after NTBC withdrawal) at the expense of their unedited counterparts [46, 47, 49]. Liver histology revealed that liver damage is substantially less severe in the $s g H p d 1$ - and $s g H p d 2$-treated mice compared to $F a h^{\text {mut } / m u t}$ mice injected with PBS, as indicated by the smaller numbers of multinucleated hepatocytes compared to PBS-injected mice (Additional file 1: Figure S3).

In vivo genome editing by NmeCas9 delivered by a rAAV vector

Although plasmid hydrodynamic injections can generate indels, therapeutic development will require less invasive delivery strategies, such as rAAV. To this end, all-in-one AAV-sgRNA-hNmeCas9 plasmids were packaged in hepatocyte-tropic AAV8 capsids to target Pcsk9 (sgPcsk9) and Rosa26 (sgRosa26) (Fig. 1b) [51, 52]. Pcsk9 and Rosa26 
were used in part to enable NmeCas9 AAV delivery to be benchmarked with that of other Cas9 orthologs delivered similarly and targeted to the same loci [18]. Vectors were administered into C57BL/6 mice via tail vein (Fig. 3a). We monitored cholesterol level in the serum and measured PCSK9 protein and indel frequencies in the liver tissues 25 and 50 days post injection.

Using a colorimetric endpoint assay, we determined that the circulating serum cholesterol level in the sgPcsk 9 mice decreased significantly $(p<0.001)$ compared to the PBS and sgRosa26 mice at 25 and 50 days post injection (Fig. 3b). Targeted deep-sequencing analyses at Pcsk9 and Rosa26 target sites revealed very efficient indels of $35 \%$ and $55 \%$, respectively, at 50 days post vector administration (Fig. 3c). Additionally, one mouse of each group was euthanized at 14 days post injection and revealed on-target indel efficiencies of 37\% and $46 \%$ at Pcsk9 and Rosa26, respectively (Fig. 3c). As expected, PCSK9 protein levels in the livers of sgPcsk9 mice were substantially reduced compared to the mice injected with PBS and sgRosa26 (Fig. 3d). The efficient editing, PCSK9 reduction, and diminished serum cholesterol indicate the successful delivery and activity of NmeCas9 at the Pcsk9 locus.

SpyCas9 delivered by viral vectors is known to elicit host immune responses $[19,53]$. To investigate if the mice injected with AAV8-sgRNA-hNmeCas9 generate anti-NmeCas9 antibodies, we used sera from the treated animals to perform IgG1 ELISA. Our results show that NmeCas9 elicits a humoral response in these animals (Additional file 1: Figure S4). Despite the presence of an immune response, NmeCas9 delivered by rAAV is highly functional in vivo, with no apparent signs of abnormalities or liver damage (Additional file 1: Figure S5).

\section{NmeCas9 is highly specific in vivo}

A significant concern in therapeutic CRISPR/Cas9 genome editing is the possibility of off-target edits. We and others have found that wild-type NmeCas9 is a naturally high-accuracy genome editing platform in cultured mammalian cells [32] (Amrani et al., manuscript in revision). To determine if NmeCas9 maintains its minimal off-targeting profile in mouse cells and in vivo, we screened for off-target sites in the mouse genome using

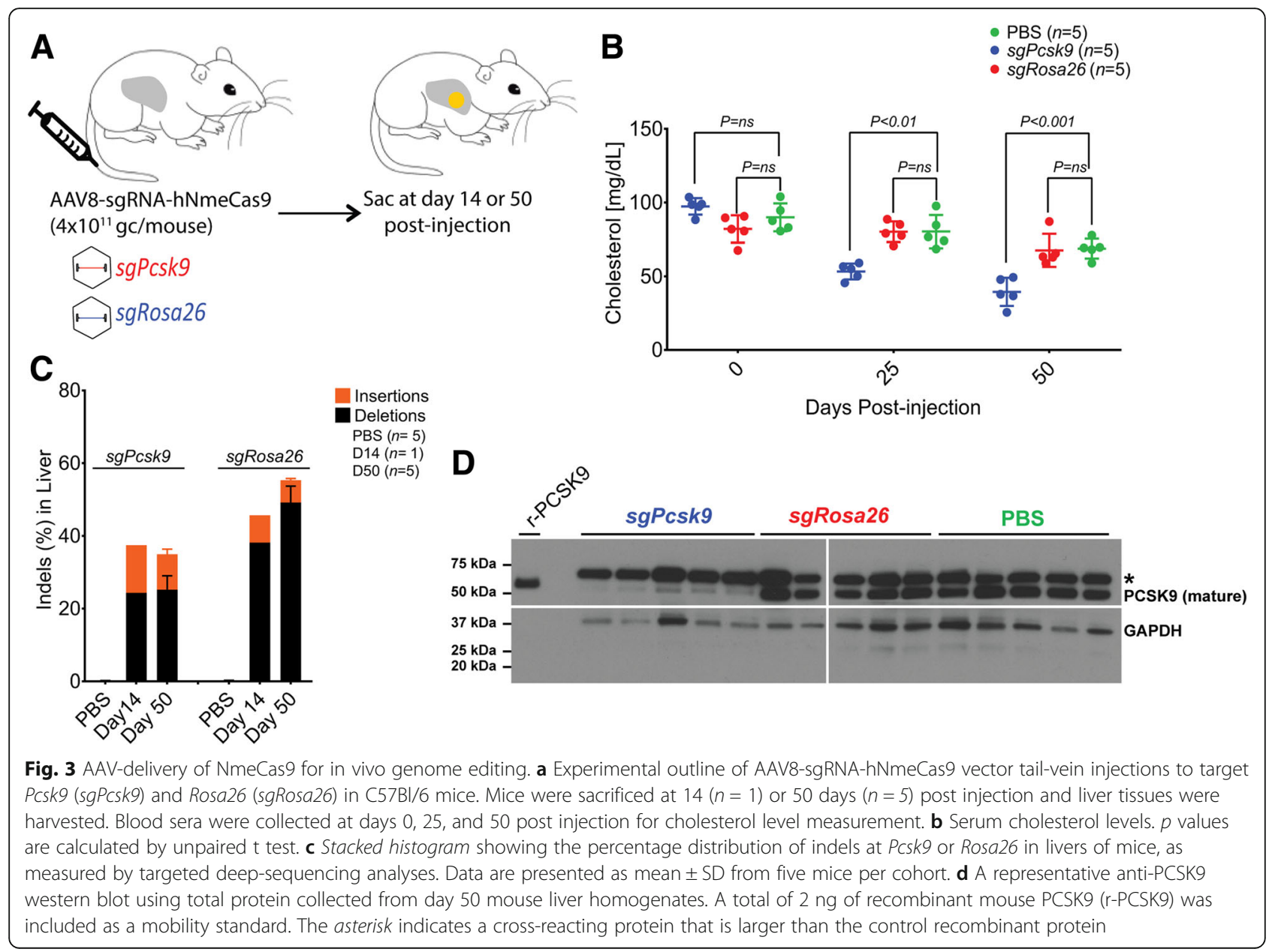


genome-wide, unbiased identification of DSBs enabled by sequencing (GUIDE-seq) [22]. Hepa1-6 cells were transfected with sgPcsk9, sgRosa26, sgHpd1, and sgHpd2 all-in-one AAV-sgRNA-hNmeCas9 plasmids and the resulting genomic DNA was subjected to GUIDE-seq analysis. Consistent with our previous observations in human cells (Amrani et al., manuscript in revision), GUIDE-seq revealed very few off-target (OT) sites in the mouse genome. Four potential OT sites were identified for sgPcsk9 and another six for sgRosa26. We were unable to detect off-target edits with $s g H p d 1$ and $s g H p d 2$ (Fig. 4a), thus reinforcing our previous observation that NmeCas 9 is often intrinsically hyper-accurate (Amrani et al., manuscript in revision).

Several of the putative OT sites for sgPcsk9 and sgRosa26 lack the NmeCas9 PAM preferences $\left(\mathrm{N}_{4} \mathrm{GATT}, \mathrm{N}_{4} \mathrm{GCTT}\right.$, $\mathrm{N}_{4}$ GTTT, $\mathrm{N}_{4}$ GACT, $\mathrm{N}_{4}$ GATA, $\mathrm{N}_{4}$ GTCT, and $\mathrm{N}_{4}$ GACA) (Fig. 4b) and may therefore represent background. To validate these OT sites, we performed targeted deep sequencing using genomic DNA from Hepa1-6 cells. By this more sensitive readout, indels were undetectable above background at all these OT sites except OT1 of Pcsk9, which had an indel frequency $<2 \%$ (Fig. $4 \mathrm{~b}$ ). To validate NmeCas9's high fidelity in vivo, we measured indel formation at these OT sites in liver genomic DNA from the AAV8-NmeCas9-treated, sgPcsk9-targeted, and sgRosa26-targeted mice. We found little or no detectable off-target editing in mice liver sacrificed at 14 days at all sites except sgPcsk9 OT1, which exhibited $<2 \%$ lesion efficiency (Fig. 4c). More importantly, this level of OT editing stayed below $<2 \%$ even after 50 days and also remained either undetectable or very low for all other candidate OT sites. These results suggested that extended (50 days) expression of NmeCas9 in vivo does not compromise its targeting fidelity (Fig. 4c).

\section{Discussion}

\section{All-in-one rAAV delivery of hNmeCas9}

Compared to transcription activator-like effector nucleases (TALENs) and Zinc-finger nucleases (ZFNs), Cas9s are distinguished by their flexibility and versatility [1]. Such characteristics make them ideal for driving the field of genome engineering forward. Over the past few years, CRISPR-Cas9 has been used to enhance products in agriculture, food, and industry, in addition to the promising applications in gene therapy and personalized medicine [54]. Despite the diversity of Class 2 CRISPR systems that have been described, only a handful of them have been
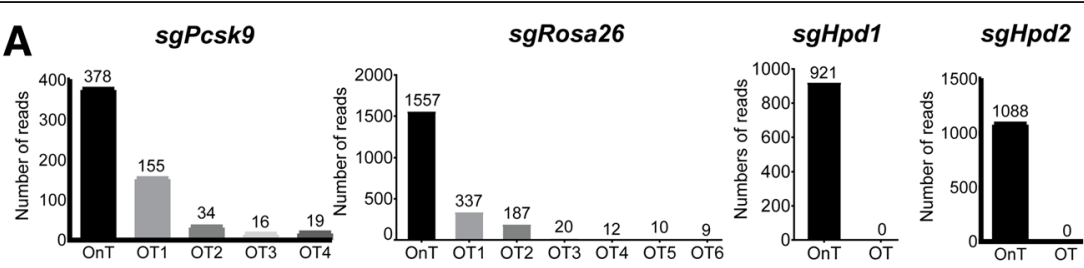

B
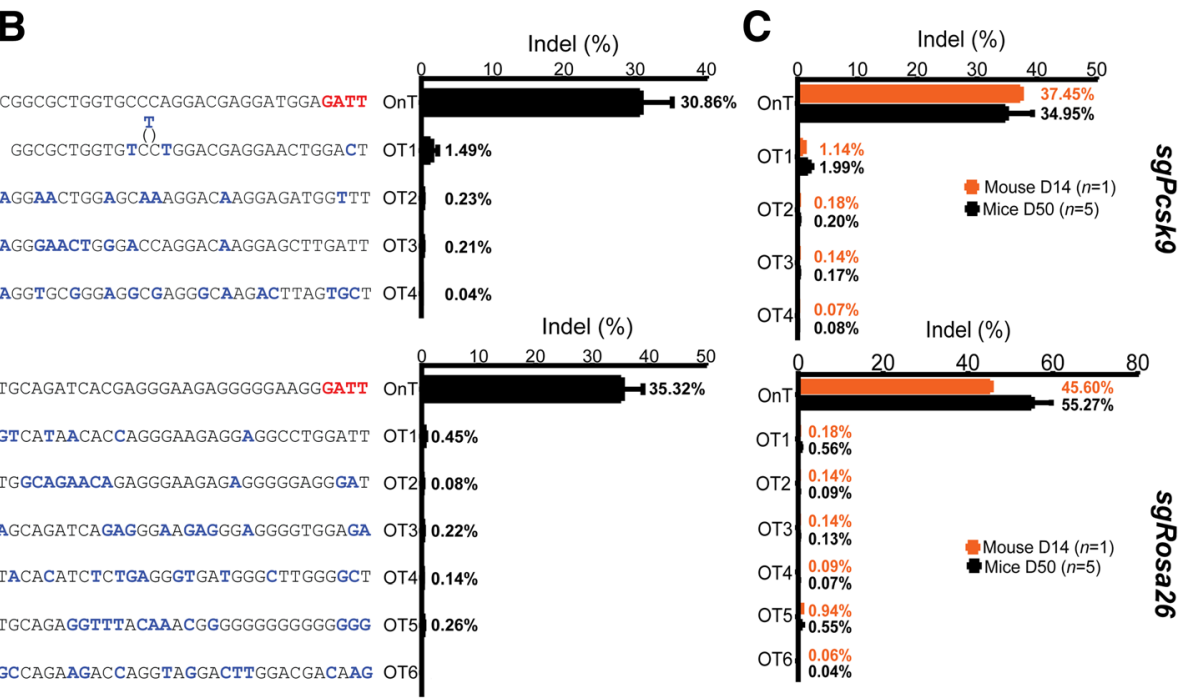

Fig. 4 GUIDE-seq genome-wide specificities of NmeCas9. a Number of GUIDE-seq reads for the on-target (OnT) and off-target (OT) sites. b Targeted deep sequencing to measure the lesion rates at each of the OT sites in Hepa1-6 cells. The mismatches of each OT site with the OnT protospacers is highlighted (blue). Data are presented as mean \pm SD from three biological replicates. c Targeted deep sequencing to measure the lesion rates at each of the OT sites using genomic DNA obtained from mice injected with all-in-one AAV8-sgRNA-hNmeCas9 sgPcsk9 and sgRosa26 and sacrificed at day 14 (D14) or day 50 (D50) post injection. Data are presented as mean \pm SD 
developed and validated for genome editing in vivo. In this study, we have shown that NmeCas9 is a compact, high-fidelity Cas9 that can be considered for future in vivo genome editing applications using all-in-one rAAV. Its unique PAM enables editing at additional targets that are inaccessible to the other two compact, all-in-one rAAV-validated orthologs (SauCas9 and CjeCas9).

\section{Therapeutic gene correction for hereditary Tyrosinemia type 1 by metabolic pathway reprograming}

Patients with mutations in the HPD gene are considered to have Type III Tyrosinemia and exhibit high level of tyrosine in blood, but otherwise appear to be largely asymptomatic $[55,56]$. HPD acts upstream of FAH in the tyrosine catabolism pathway and $H p d$ disruption ameliorates HT-I symptoms by preventing the toxic metabolite build-up that results from loss of FAH. Structural analyses of HPD reveal that the catalytic domain of the HPD enzyme is located at the C-terminus of the enzyme and encoded by exon 13 and 14 [57]. Thus, frameshift-inducing indels upstream of exon 13 should render the enzyme inactive. We used this context to demonstrate that $\mathrm{Hpd}$ inactivation by hydrodynamic injection of NmeCas9 plasmid is a viable approach to rescue HT-I mice. NmeCas9 can edit sites carrying several different PAMs $\left(\mathrm{N}_{4}\right.$ GATT [consensus], $\mathrm{N}_{4}$ GCTT, $\mathrm{N}_{4}$ GTTT, $\mathrm{N}_{4}$ GACT, $\mathrm{N}_{4}$ GATA, $\mathrm{N}_{4}$ GTCT, and $\mathrm{N}_{4}$ GACA) (Amrani et al., manuscript in revision). Our $H p d$ editing experiments confirmed one of the variant PAMs in vivo with the $s g H p d 2$ guide, which targets a site with a $\mathrm{N}_{4}$ GACT PAM.

\section{Efficient, accurate NmeCas9 genome editing with rAAV delivery}

To achieve targeted delivery of NmeCas9 to various tissues in vivo, rAAV vectors are a promising delivery platform due to the compact size of NmeCas9 transgene, which allows the delivery of NmeCas9 and its guide in an all-in-one format. We have validated this approach for the targeting of Pcsk 9 and Rosa 26 genes in adult mice, with efficient editing observed even at 14 days post injection. As observed previously in cultured cells [32] (Amrani et al., manuscript in revision), NmeCas9 is intrinsically accurate, even without the extensive engineering that was required to reduce off-targeting by SpyCas9 [21-23]. We performed side-by-side comparisons of NmeCas9 OT editing in cultured cells and in vivo by targeted deep sequencing and we found that off-targeting is minimal in both settings. Editing at the sgPcsk9 OT1 site (within an unannotated locus) was the highest detectable at $\sim 2 \%$. Despite these promising results, more extensive and long-term studies, including in larger animals, will be needed to fully understand the long-term effects of Cas9 expression in tissues, as well as the development of approaches that clear viral vectors after editing is complete.

\section{Conclusions}

We demonstrate that NmeCas9 is amenable to in vivo genome editing using the highly desirable all-in-one rAAV platform. With its unique PAM preferences and high fidelity, this all-in-one AAV-sgRNA-hNeCas9 can be applied to a range of genome editing purposes in vivo. We anticipate that successful in vivo delivery of this accurate and effective Cas9 can advance therapeutic editing in humans.

\section{Methods}

Construction of all-in-one AAV-sgRNA-hNMeCas9 plasmid and rAAV vector production

The human-codon-optimized NmeCas9 gene under the control of the U1a promoter and a sgRNA cassette driven by the U6 promoter were cloned into an AAV2 plasmid backbone. The NmeCas9 ORF was flanked by four nuclear localization signals - two on each terminus - in addition to a triple-HA epitope tag. This plasmid is available through Addgene (plasmid ID 112139). Oligonucleotides with spacer sequences targeting Hpd, Pcsk9, and Rosa26 were inserted into the sgRNA cassette by ligation into a SapI cloning site (Additional file 2).

AAV vector production was performed at the Horae Gene Therapy Center at the University of Massachusetts Medical School. Briefly, plasmids were packaged in AAV8 capsid by triple-plasmid transfection in HEK293 cells and purified by sedimentation as previously described [58].

The off-target profiles of these spacers were predicted computationally using the Bioconductor package CRISPRseek. Search parameters were adapted to NmeCas9 settings as described previous (Amrani et al., manuscript in revision): gRNA.size $=24$, PAM $=$ "NNNNGATT," PAM.size $=8$, RNA.PAM.pattern $=$ "NNNNGNNN\$," weights $=$ $\mathrm{c}(0,0,0,0,0,0,0.014,0,0,0.395,0.317,0,0.389,0.079$, $0.445,0.508,0.613,0.851,0.732,0.828,0.615,0.804,0.685$, 0.583), max.mismatch $=6$, allowed.mismatch.PAM $=7$, topN $=10,000$, min.score $=0$.

\section{Cell culture and transfection}

Mouse Hepa1-6 hepatoma cells were cultured in DMEM with $10 \%$ fetal bovine serum and $1 \%$ Penicillin/Streptomycin (Gibco) in a $37^{\circ} \mathrm{C}$ incubator with $5 \% \mathrm{CO}_{2}$. Transient transfections of Hepa1-6 cells were performed using Lipofectamine LTX. For transient transfection, approximately $1 \times 10^{5}$ cells per well were cultured in 24-well plates $24 \mathrm{~h}$ before transfection. Each well was transfected with 500-ng all-in-one AAV-sgRNA-hNmeCas9 plasmid, using Lipofectamine LTX with Plus Reagent (Invitrogen) according to the manufacturer's protocol.

\section{DNA isolation from cells and liver tissue}

Genomic DNA isolation from cells was performed $72 \mathrm{~h}$ post transfection using DNeasy Blood and Tissue kit 
(Qiagen) following the manufacturer's protocol. Mice were sacrificed and liver tissues were collected 10 days post hydrodynamic injection or 14 and 50 days post tail-vein rAAV injection. Genomic DNA was isolated using DNeasy Blood and Tissue kit (Qiagen) according to the manufacturer's protocol.

\section{GUIDE-seq}

GUIDE-seq analysis was performed as previously described [22]. Briefly, 7.5 pmol of annealed GUIDE-seq oligonucleotides and $500 \mathrm{ng}$ of all-in-one AAV-sgRNA-hNmeCas9 plasmids targeting Pcsk9, Rosa26, and Hpd were transfected into $1 \times 10^{5}$ Hepa1-6 cells using Lipofectamine LTX with Plus Reagent (Invitrogen). At $72 \mathrm{~h}$ post transfection, genomic DNA was extracted using a DNeasy Blood and Tissue kit (Qiagen) per manufacturer protocol. Library preparations, deep sequencing, and reads analysis were performed as previously described $[59,60]$. The Bioconductor package GUIDEseq was used for off-target analysis as described previously using maximum allowed mismatch of $10 \mathrm{nt}$ between the guide and target DNA [59]. For read alignment, mouse mm10 was used as a reference genome.

\section{Indel analysis}

TIDE primers were designed $\sim 700$ bp apart, with the forward primer at $\sim 200 \mathrm{bp}$ upstream of the cleavage site. A total of 50 ng of genomic DNA was used for PCR amplification with High Fidelity 2X PCR Master Mix (New England Biolabs). For TIDE analysis, $30 \mu \mathrm{L}$ of a PCR product was purified using QIAquick PCR Purification Kit (Qiagen) and sent for Sanger sequencing using the TIDE forward primer (Additional file 3). Indel values were obtained using the TIDE web tool (https://tide-cal culator.nki.nl/) as described previously [41].

Targeted deep-sequencing analysis was performed for Hepa1-6 cells and mouse liver gDNA using a two-step PCR amplification approach as described previously [60] (Amrani et al., manuscript in revision). Briefly, in the first PCR step, on-target or off-target locus-specific primers were used to amplify the editing site using Phusion High Fidelity DNA Polymerase (New England Biolabs) with a $65^{\circ}$ $\mathrm{C}$ annealing temperature. The primer ends contained sequences complementary to Illumina TruSeq adaptor sequences (Additional file 3). In the second-step PCR, equimolar amounts of DNA were amplified with a universal forward primer and an indexed reverse primer using Phusion High Fidelity DNA Polymerase $\left(98^{\circ} \mathrm{C}, 15 \mathrm{~s} ; 61^{\circ} \mathrm{C}\right.$, $25 \mathrm{~s} ; 72^{\circ} \mathrm{C}, 18 \mathrm{~s}$; nine cycles) to ligate the TruSeq adaptors. The resultant amplicons were separated in a $2.5 \%$ agarose gel and the corresponding $\sim 250$-bp product bands were extracted using Monarch DNA Gel Extraction Kit (New England Biolabs).

The libraries were then sequenced on an Illumina MiSeq in paired-end mode with a read length of $150 \mathrm{bp}$.
To analyze genome editing outcomes at genomic sites, the command line utilities of CRISPResso were used [61]. Input parameters were adjusted to filter low-quality reads (-q 30 -s 20). Furthermore, the background was determined using the control sample (no guide) and subtracted from the experimental samples. The resulting indel frequencies, sizes, and distributions were then plotted using Graphpad PRISM.

\section{Animals and liver tissue processing}

For hydrodynamic injections, $2.5 \mathrm{~mL}$ of $30 \mu \mathrm{g}$ of endotoxin-free AAV-sgRNA-hNmeCas9 plasmid targeting Pcsk9 or $2.5 \mathrm{~mL}$ PBS was injected by tail vein into 9to 18 -week-old female C57BL/6 mice. Mice were euthanized 10 days later and liver tissue was harvested. For the AAV8 vector injections, 12 - to 16 -week-old female C57BL/6 mice were injected with $4 \times 10^{11}$ genome copies per mouse via tail vein, using vectors targeting Pcsk 9 or Rosa26. Mice were sacrificed 14 and 50 days after vector administration and liver tissues were collected for analysis.

For Hpd targeting, $2 \mathrm{~mL}$ PBS or $2 \mathrm{~mL}$ of $30 \mu \mathrm{g}$ of endotoxin-free AAV-sgRNA-hNmeCas9 plasmid was administered into 15- to 21-week-old Type 1 Tyrosinemia Fah knockout mice $\left(F a h^{\text {neo }}\right)$ via tail vein. The encoded sgRNAs targeted sites in exon 8 (sgHpd1) or exon 11 (sgHpd2). The HT1 homozygous mice with the $F a h^{\text {neo }}$ allele in a 129 background were kindly provided by Dr. Markus Grompe [50]. The HT1 mice were fed with $10 \mathrm{mg} / \mathrm{L}$ NTBC (2-(2-nitro-4-trifluoromethylbenzoyl)-1,3-cyclohexanedione) (Sigma-Aldrich, Cat. No. PHR1731-1G) in drinking water when indicated. Both sexes were used in these experiments. Mice were maintained on NTBC water for seven days post injection and then switched to normal water. Body weight was monitored every 1-3 days. The PBS-injected control mice were sacrificed when they became moribund after losing $20 \%$ of their body weight after removal from NTBC treatment.

Mice were euthanized according to our protocol and liver tissue was sliced and fragments stored at $-80^{\circ} \mathrm{C}$. Some liver tissues were fixed in $4 \%$ formalin overnight, embedded in paraffin, sectioned and stained with hematoxylin and eosin (H\&E).

\section{Serum analysis}

Blood $(\sim 200 \mu \mathrm{L})$ was drawn from the facial vein at 0 , 25 , and 50 days post vector administration. Serum was isolated using a serum separator (BD, Cat. No. 365967) and stored under $-80^{\circ} \mathrm{C}$ until assay.

Serum cholesterol levels were measured by Infinity ${ }^{\mathrm{mm}}$ colorimetric endpoint assay (Thermo-Scientific) following the manufacturer's protocol. Briefly, serial dilutions of Data-Cal ${ }^{\mathrm{ma}}$ Chemistry Calibrator were prepared in PBS. In a 96-well plate, $2 \mu \mathrm{L}$ of mice sera or calibrator 
dilution was mixed with $200 \mu \mathrm{L}$ of Infinity ${ }^{\text {mit }}$ cholesterol liquid reagent, then incubated at $37^{\circ} \mathrm{C}$ for $5 \mathrm{~min}$. The absorbance was measured at $500 \mathrm{~nm}$ using a BioTek Synergy HT microplate reader.

\section{Western blot}

Liver tissue fractions were ground and resuspended in $150 \mu \mathrm{L}$ of RIPA lysis buffer. Total protein content was estimated by Pierce ${ }^{\mathrm{mt}}$ BCA Protein Assay Kit (Thermo-Scientific) following the manufacturer's protocol. A total of $20 \mu \mathrm{g}$ of protein from tissue or $2 \mathrm{ng}$ of Recombinant Mouse Proprotein Convertase 9/PCSK9 Protein (R\&D Systems, 9258-SE-020) were loaded onto a 4-20\% Mini-PROTEAN $^{\circ}$ TGX $^{\mathrm{m}}$ Precast Gel (Bio-Rad). The separated bands were transferred onto PVDF membrane and blocked with 5\% Blocking-Grade Blocker solution (Bio-Rad) for $2 \mathrm{~h}$ at room temperature. Membranes were incubated with rabbit anti-GAPDH (Abcam ab9485, 1:2000) or goat anti-PCSK9 (R\&D Systems AF3985, 1:400) antibodies overnight at $4{ }^{\circ} \mathrm{C}$. Membranes were washed five times in TBST and incubated with horseradish peroxidase (HRP)-conjugated goat anti-rabbit (Bio-Rad 1,706,515, 1:4000) and donkey anti-goat (R\&D Systems HAF109, 1:2000) secondary antibodies for $2 \mathrm{~h}$ at room temperature. The membranes were washed five times in TBST and visualized with Clarity ${ }^{\mathrm{mm}}$ western ECL substrate (Bio-Rad) using an M35A X-OMAT Processor (Kodak).

\section{Humoral immune response}

Humoral IgG1 immune response to NmeCas9 was measured by ELISA (Bethyl; Mouse IgG1 ELISA Kit, E99105) following manufacturer's protocol with a few modifications. Briefly, expression and three-step purification of NmeCas9 and SpyCas9 was performed as previously described [4]. A total of $0.5 \mu \mathrm{g}$ of recombinant NmeCas9 or SpyCas9 proteins suspended in $1 \times$ coating buffer (Bethyl) were used to coat 96-well plates (Corning) and incubated for $12 \mathrm{~h}$ at $4{ }^{\circ} \mathrm{C}$ with shaking. The wells were washed three times while shaking for 5 min using $1 \times$ Wash Buffer. Plates were blocked with $1 \times$ BSA Blocking Solution (Bethyl) for $2 \mathrm{~h}$ at room temperature, then washed three times. Serum samples were diluted 1:40 using PBS and added to each well in duplicate. After incubating the samples at $4{ }^{\circ} \mathrm{C}$ for $5 \mathrm{~h}$, the plates were washed $3 \times$ times for $5 \mathrm{~min}$ and $100 \mu \mathrm{L}$ of biotinylated anti-mouse IgG1 antibody (Bethyl; 1: 100,000 in 1 x BSA Blocking Solution) was added to each well. After incubating for $1 \mathrm{~h}$ at room temperature, the plates were washed four times and $100 \mu \mathrm{L}$ of TMB Substrate was added to each well. The plates were allowed to develop in the dark for $20 \mathrm{~min}$ at room temperature and $100 \mu \mathrm{L}$ of ELISA Stop Solution was then added per well. Following the development of the yellow solution, absorbance was recorded at $450 \mathrm{~nm}$ using a BioTek Synergy HT microplate reader.

\section{Additional files}

\begin{abstract}
Additional file 1: Figure S1. Genome-wide computational prediction of NmeCas9 off-target sites using CRISPRseek with the $\mathrm{N}_{4} G N_{3}$ PAM. Search parameters were set to identify sites with up to six mismatches to the spacer sequence. The total number of detected off-target sites for each protospacer is indicated. Figure S2. Stacked histogram showing the percentage distribution of indels obtained by TIDE after AAV-sgRNAhNmeCas9 plasmids transfections in Hepa1-6. Error bars represent three independent experiments. Data are presented as mean \pm SD. Figure S3. H\&E staining from wild-type $\left(\mathrm{Fah}^{+/+}\right)$mouse, and HT-I mice $\left(\mathrm{Fah}^{-/-}\right)$ injected with PBS or AAV-sgRNA-hNmeCas9 plasmids sgHpd1 or sgHpd2. Scale bar is $20 \mu \mathrm{m}$. Figure S4. Humoral lgG1 immune response to NmeCas 9 in vivo. Serum collected at day 50 post injection with all-in-one AAV8-sgRNA-hNmeCas9 sgPcsk9 and sgRosa26. Serum antibodies reacted against NmeCas9 protein (left) or SpyCas9 protein (right). Figure S5. H\&E staining from PBS, sgPcsk9, and sgRosa26 AAV8 injected mice. Scale bar is $20 \mu \mathrm{m}$. (PDF $713 \mathrm{~kb}$ )
\end{abstract}

Additional file 2: Nucleotide sequence of all-in-one AAV-sgRNAhNmeCas9 Plasmid. (PDF 59 kb)

Additional file 3: Contains Protospacer sequences, TIDE Primers sequences, and Deep-sequencing Primers sequences. (PDF 69 kb)

\section{Abbreviations}

AAV: Adeno-associated virus; bp: Base pair; Cas: CRISPR-associated; CjeCas9: Campylobacter jejuni Cas9; CRISPR: Clustered, regularly interspaced, short palindromic repeats; crRNAs: CRISPR RNAs; dCas9: "Dead" Cas9; DSB: Double-strand breaks; dsODN: Double-stranded oligodeoxynucleotide; FAH: Fumarylacetoacetate hydroxylase; GUIDE-seq: Genome-wide unbiased identification of double strand breaks enabled by sequencing; HDR: Homologydirected repair; HPD: Hydroxyphenylpyruvate dioxygenase; HTI: Hereditary tyrosinemia type I; IRD: Inherited retinal disease; LDL: Low-density lipoprotein; MGEs: Mobile genetic elements; NHEJ: Non-homologous end joining; NLS: Nuclear localization signal; NmeCas9: Neisseria meningitidis (strain 8013) Cas9; NTBC: 2-(2-Nitro-4-trifluoromethylbenzoyl) -1,3-cyclohexanedione; NTS: NmeCas9 target site; PAM: Protospacer adjacent motif; PCSK9: Proprotein convertase subtilisin/kexin type 9; rAAV: Recombinant adeno-associated virus; RNP: Ribonucleoprotein; SauCas9: Staphylococcus aureus Cas9; sgRNA: Singleguide RNA; SpyCas9: Streptococcus pyogenes Cas9; TALENs: Transcription activator-like effector nucleases; TIDE: Tracking of indels by decomposition; tracrRNA: Trans-acting CRISPR RNA; ZFNs: Zinc-finger nucleases

\section{Acknowledgements}

We thank Haiwei Mou and Yueying Cao for their assistance with mouse injections and Xin Gao for his assistance in designing of deep sequencing and GUIDE-seq libraries. We also thank the UMMS Viral Vector Core for AAV packaging services and the UMMS Deep Sequencing Core for sequencing. We are grateful to Guangping Gao, Scot Wolfe, and all members of the Xue and Sontheimer labs for their discussions, advice, and helpful feedback.

\section{Funding}

WX was supported by the NIH (DP2HL137167 and P01HL131471), the Lung Cancer Research Foundation, the ALS Association, the American Cancer Society (129056-RSG-16-093), and Hyundai Hope on Wheels. EJS was supported by the NIH (R01GM1115911 to SAW and EJS).

\section{Availability of data and materials}

The deep sequencing data from this study are available at NCBI Sequence Read Archive (SRA) under accession number SRP150799 [62]. All-in-one AAVsgRNA-hNmeCas9 plasmid is available through Addgene (plasmid ID 112139).

\section{Authors' contributions}

$\mathrm{RI}$ and EJS initiated the study; RI, CS, WX, and EJS designed experiments. RI designed, constructed, and validated all-in-one AAV-sgRNA-hNmeCas9 plasmids in cells, prepared GUIDE-seq, targeted lesion libraries from cultured cells and mouse tissues, and analyzed in vivo-edited samples. CS performed mouse manipulations including plasmid hydrodynamic injections, AAV performed injections as well as serum and animal tissue collection. NA assisted with construct design and GUIDE-seq library preparation. AM 
analyzed deep sequencing and GUIDE-seq datasets. RI, AM, and EJS wrote the manuscript and all authors edited the manuscript. All authors read and approved the final manuscript.

\section{Ethics approval}

All animal procedures were reviewed and approved by The Institutional Animal Care and Use Committee (IACUC) at University of Massachusetts Medical School (IACUC approval number 2490).

\section{Consent for publication}

Not applicable.

\section{Competing interests}

EJS is a co-founder and advisor of Intellia Therapeutics, Inc

\section{Publisher's Note}

Springer Nature remains neutral with regard to jurisdictional claims in published maps and institutional affiliations.

\section{Received: 20 July 2018 Accepted: 22 August 2018 Published online: 19 September 2018}

\section{References}

1. Komor AC, Badran AH, Liu DR. CRISPR-based technologies for the manipulation of eukaryotic genomes. Cell. 2017;168:20-36.

2. Sontheimer EJ, Barrangou R. The bacterial origins of the CRISPR genomeediting revolution. Hum Gene Ther. 2015;26:413-24.

3. Hille F, Richter H, Wong SP, Bratovic M, Ressel S, Charpentier E. The biology of CRISPR-Cas: backward and forward. Cell. 2018;172:1239-59.

4. Jinek M, Chylinski K, Fonfara I, Hauer M, Doudna JA, Charpentier E. A programmable dual-RNA-guided DNA endonuclease in adaptive bacterial immunity. Science. 2012;337:816-21.

5. Yin H, Kauffman KJ, Anderson DG. Delivery technologies for genome editing. Nat Rev Drug Discov. 2017;16:387-99.

6. Wong JKL, Mohseni R, Hamidieh AA, MacLaren RE, Habib N, Seifalian AM. Limitations in clinical translation of nanoparticle-based gene therapy. Trends Biotechnol. 2017:35:1124-5.

7. Lee K, Conboy M, Park HM, Jiang F, Kim HJ, Dewitt MA, et al. Nanoparticle delivery of Cas9 ribonucleoprotein and donor DNA in vivo induces homology-directed DNA repair. Nat Biomed Eng. 2017;1:889-901.

8. Yin H, Song C-Q, Suresh S, Wu Q, Walsh S, Rhym LH, et al. Structure-guided chemical modification of guide RNA enables potent non-viral in vivo genome editing. Nat Biotechnol. 2017;35:1179

9. Grieger JC, Samulski RJ. Adeno-associated virus vectorology, manufacturing, and clinical applications. Methods Enzymol. 2012;507:229-54

10. Vassalli G, Bueler H, Dudler J, von Segesser LK, Kappenberger L. Adenoassociated virus (AAV) vectors achieve prolonged transgene expression in mouse myocardium and arteries in vivo: a comparative study with adenovirus vectors. Int J Cardiol. 2003:90:229-38.

11. Zincarelli C, Soltys S, Rengo G, Rabinowitz JE. Analysis of AAV serotypes 1-9 mediated gene expression and tropism in mice after systemic injection. Mol Ther. 2008;16:1073-80.

12. Penaud-Budloo M, Le Guiner C, Nowrouzi A, Toromanoff A, Cherel $Y$, Chenuaud $P$, et al. Adeno-associated virus vector genomes persist as episomal chromatin in primate muscle. J Virol. 2008;82:7875-85.

13. Nayak S, Herzog RW. Progress and prospects: immune responses to vira vectors. Gene Ther. 2010;17:295-304.

14. Sack BK, Herzog RW. Evading the immune response upon in vivo gene therapy with viral vectors. Curr Opin Mol Ther. 2009:11:493-503.

15. Keeler AM, ElMallah MK, Flotte TR. Gene therapy 2017: progress and future directions. Clin Transl Sci. 2017:10:242-8.

16. Bennett J, Wellman J, Marshall KA, McCague S, Ashtari M, DiStefano-Pappas $J$, et al. Safety and durability of effect of contralateral-eye administration of AAV2 gene therapy in patients with childhood-onset blindness caused by RPE65 mutations: a follow-on phase 1 trial. Lancet. 2016;388:661-72.

17. Swiech L, Heidenreich M, Banerjee A, Habib N, Li Y, Trombetta J, et al. In vivo interrogation of gene function in the mammalian brain using CRISPRCas9. Nat Biotechnol. 2015:33:102-6.

18. Ran FA, Cong L, Yan WX, Scott DA, Gootenberg JS, Kriz AJ, et al. In vivo genome editing using Staphylococcus aureus Cas9. Nature. 2015;520: 186-91.
19. Chew WL, Tabebordbar M, Cheng JK, Mali P, Wu EY, Ng AH, et al. A multifunctional AAV-CRISPR-Cas9 and its host response. Nat Methods. 2016; 13:868-74.

20. Kim E, Koo T, Park SW, Kim D, Kim K, Cho HY, et al. In vivo genome editing with a small Cas9 orthologue derived from campylobacter jejuni. Nat Commun. 2017:8:14500

21. Bolukbasi MF, Gupta A, Wolfe SA. Creating and evaluating accurate CRISPRCas9 scalpels for genomic surgery. Nat Methods. 2016;13:41-50.

22. Tsai SQ, Joung JK. Defining and improving the genome-wide specificities of CRISPR-Cas9 nucleases. Nat Rev Genet. 2016;17:300-12.

23. Tycko J, Myer VE, Hsu PD. Methods for optimizing CRISPR-Cas9 genome editing specificity. Mol Cell. 2016;63:355-70.

24. Wu Z, Yang $H$, Colosi P. Effect of genome size on AAV vector packaging. Mol Ther. 2010;18:80-6.

25. Wright AV, Sternberg SH, Taylor DW, Staahl BT, Bardales JA, Kornfeld JE, et al. Rational design of a split-Cas9 enzyme complex. Proc Natl Acad Sci U S A. 2015;112:2984-9.

26. Zetsche B, Volz SE, Zhang F. A split-Cas9 architecture for inducible genome editing and transcription modulation. Nat Biotechnol. 2015;33:139-42.

27. Truong DJ, Kuhner K, Kuhn R, Werfel S, Engelhardt S, Wurst W, et al. Development of an intein-mediated split-Cas9 system for gene therapy. Nucleic Acids Res. 2015;43:6450-8.

28. Suzuki K, Tsunekawa Y, Hernandez-Benitez R, Wu J, Zhu J, Kim EJ, et al. In vivo genome editing via CRISPR/Cas9 mediated homology-independent targeted integration. Nature. 2016;540:144-9.

29. Mir A, Edraki A, Lee J, Sontheimer EJ. Type II-C CRISPR-Cas9 biology, mechanism, and application. ACS Chem Biol. 2018;13:357-65.

30. Friedland $A E$, Baral $R$, Singhal $P$, Loveluck $K$, Shen $S$, Sanchez M, et al. Characterization of Staphylococcus aureus Cas9: a smaller Cas9 for all-in-one adeno-associated virus delivery and paired nickase applications. Genome Biol. 2015;16:257.

31. Esvelt KM, Mali P, Braff JL, Moosburner M, Yaung SJ, Church GM. Orthogonal Cas9 proteins for RNA-guided gene regulation and editing. Nat Methods. 2013;10:1116-21.

32. Lee CM, Cradick TJ, Bao G. The Neisseria meningitidis CRISPR-Cas9 system enables specific genome editing in mammalian cells. Mol Ther. 2016;24:645-54.

33. Hou Z, Zhang Y, Propson NE, Howden SE, Chu LF, Sontheimer EJ, et al. Efficient genome engineering in human pluripotent stem cells using Cas9 from Neisseria meningitidis. Proc Natl Acad Sci U S A. 2013;110:15644-9.

34. Pawluk A, Amrani N, Zhang Y, Garcia B, Hidalgo-Reyes $Y$, Lee J, et al. Naturally occurring off-switches for CRISPR-Cas9. Cell. 2016;167:1829-38.

35. Pankowicz FP, Barzi M, Legras X, Hubert L, Mi T, Tomolonis JA, et al. Reprogramming metabolic pathways in vivo with CRISPR/Cas9 genome editing to treat hereditary tyrosinaemia. Nat Commun. 2016;7:12642.

36. Mousavi SA, Berge KE, Leren TP. The unique role of proprotein convertase subtilisin/kexin 9 in cholesterol homeostasis. J Intern Med. 2009:266:507-19.

37. Lagace TA. PCSK9 and LDLR degradation: regulatory mechanisms in circulation and in cells. Curr Opin Lipidol. 2014:25:387-93.

38. Fitzgerald K, White S, Borodovsky A, Bettencourt BR, Strahs A, Clausen V, et al. A highly durable RNAi therapeutic inhibitor of PCSK9. N Engl J Med. 2017;376:41-51

39. Rashid S, Curtis DE, Garuti R, Anderson NN, Bashmakov Y, Ho YK, et al. Decreased plasma cholesterol and hypersensitivity to statins in mice lacking Pcsk9. Proc Natl Acad Sci U S A. 2005:102:5374-9.

40. Zhu LJ, Holmes BR, Aronin N, Brodsky MH. CRISPRseek: a bioconductor package to identify target-specific guide RNAs for CRISPR-Cas9 genomeediting systems. PLoS One. 2014;9:e108424.

41. Brinkman EK, Chen T, Amendola M, van Steensel B. Easy quantitative assessment of genome editing by sequence trace decomposition. Nucleic Acids Res. 2014:42:e168.

42. Liu F, Song Y, Liu D. Hydrodynamics-based transfection in animals by systemic administration of plasmid DNA. Gene Ther. 1999;6:1258-66.

43. Xue W, Chen S, Yin H, Tammela T, Papagiannakopoulos T, Joshi NS, et al. CRISPR-mediated direct mutation of cancer genes in the mouse liver. Nature. 2014:514:380-4.

44. Grompe M. The pathophysiology and treatment of hereditary tyrosinemia type 1. Semin Liver Dis. 2001;21:563-71.

45. Lindstedt S, Holme E, Lock EA, Hjalmarson O, Strandvik B. Treatment of hereditary tyrosinaemia type I by inhibition of 4-hydroxyphenylpyruvate dioxygenase. Lancet. 1992;340:813-7. 
46. Das AM. Clinical utility of nitisinone for the treatment of hereditary tyrosinemia type-1 (HT-1). Appl Clin Genet. 2017;10:43-8.

47. Paulk NK, Wursthorn K, Wang Z, Finegold MJ, Kay MA, Grompe M. Adenoassociated virus gene repair corrects a mouse model of hereditary tyrosinemia in vivo. Hepatology. 2010;51:1200-8.

48. Yin $\mathrm{H}$, Song $\mathrm{CQ}$, Dorkin JR, Zhu LJ, Li Y, Wu Q, et al. Therapeutic genome editing by combined viral and non-viral delivery of CRISPR system components in vivo. Nat Biotechnol. 2016;34:328-33.

49. Yin H, Xue W, Chen S, Bogorad RL, Benedetti E, Grompe M, et al. Genome editing with Cas9 in adult mice corrects a disease mutation and phenotype. Nat Biotechnol. 2014:32:551-3.

50. Grompe M, al-Dhalimy M, Finegold M, Ou CN, Burlingame T, Kennaway NG, et al. Loss of fumarylacetoacetate hydrolase is responsible for the neonatal hepatic dysfunction phenotype of lethal albino mice. Genes Dev. 1993;7: 2298-307.

51. Gao GP, Alvira MR, Wang L, Calcedo R, Johnston J, Wilson JM. Novel adenoassociated viruses from rhesus monkeys as vectors for human gene therapy. Proc Natl Acad Sci U S A. 2002;99:11854-9.

52. Nakai H, Fuess S, Storm TA, Muramatsu S, Nara Y, Kay MA. Unrestricted hepatocyte transduction with adeno-associated virus serotype 8 vectors in mice. J Virol. 2005;79:214-24.

53. Wang D, Mou H, Li S, Li Y, Hough S, Tran K, et al. Adenovirus-mediated somatic genome editing of Pten by CRISPR/Cas9 in mouse liver in spite of Cas9-specific immune responses. Hum Gene Ther. 2015:26:432-42.

54. Barrangou R, Doudna JA. Applications of CRISPR technologies in research and beyond. Nat Biotechnol. 2016;34:933-41.

55. Szymanska E, Sredzinska M, Ciara E, Piekutowska-Abramczuk D, Ploski R, Rokicki D, et al. Tyrosinemia type III in an asymptomatic girl. Mol Genet Metab Rep. 2015;5:48-50.

56. Nakamura K, Tanaka Y, Mitsubuchi H, Endo F. Animal models of tyrosinemia. J Nutr. 2007;137:1556S-60S. discussion 1573S-5S

57. Huang CW, Liu HC, Shen CP, Chen YT, Lee SJ, Lloyd MD, et al. The different catalytic roles of the metal-binding ligands in human 4-

hydroxyphenylpyruvate dioxygenase. Biochem J. 2016;473:1179-89.

58. Gao GP, Sena-Esteves M. Introducing genes into mammalian cells: viral vectors. In: Green MR, Sambrook J, editors. Molecular cloning: a laboratory manual. Volume 2. 4th ed. New York: Cold Spring Harbor Laboratory Press; 2012. p. 1209-13.

59. Zhu LJ, Lawrence M, Gupta A, Pages H, Kucukural A, Garber M, et al. GUIDEseq: a bioconductor package to analyze GUIDE-Seq datasets for CRISPR-Cas nucleases. BMC Genomics. 2017;18:379.

60. Bolukbasi MF, Gupta A, Oikemus S, Derr AG, Garber M, Brodsky MH, et al. DNA-binding-domain fusions enhance the targeting range and precision of Cas9. Nat Methods. 2015:12:1150-6.

61. Pinello L, Canver MC, Hoban MD, Orkin SH, Kohn DB, Bauer DE, et al. Analyzing CRISPR genome-editing experiments with CRISPResso. Nat Biotechnol. 2016;34:695-7.

62. Ibraheim R, Song C-Q, Mir A, Amrani N, Xue W, Sontheimer E. All-in-one adeno-associated virus delivery and genome editing by Neisseria meningitidis Cas9 in vivo NCBI Sequence Read Archive (SRA). https://www.ncbi.nlm.nih.gov/ sra/SRP150799.

\section{Ready to submit your research? Choose BMC and benefit from:}

- fast, convenient online submission

- thorough peer review by experienced researchers in your field

- rapid publication on acceptance

- support for research data, including large and complex data types

- gold Open Access which fosters wider collaboration and increased citations

- maximum visibility for your research: over $100 \mathrm{M}$ website views per year

At $\mathrm{BMC}$, research is always in progress.

Learn more biomedcentral.com/submissions 